\title{
CHEMICAL AND BIOLOGICAL DIAGNOSTICS USING FULLY INSULATED ULTRACOMPLIANT THERMAL PROBES
}

\author{
$\underline{\text { M.-H. Li }}{ }^{1}$, J.-H. Lee ${ }^{1}$, F. Cerrina ${ }^{1}$, A.K. Menon ${ }^{2}$, and Y. B. Gianchandani ${ }^{1,3}$ \\ ${ }^{1}$ Department of Electrical and Computer Engineering, University of Wisconsin, Madison, WI 53706 \\ ${ }^{2}$ Department of Biochemistry, University of Wisconsin, Madison, WI 53706 \\ ${ }^{3}$ EECS Department, University of Michigan, Ann Arbor, MI 48109
}

\begin{abstract}
This paper reports on the detection of nano-scale chemical variations in photosensitive polymers and biological variations in ovarian cancerous tumor cells that have been accomplished for the first time using scanning thermal probes that we have developed. A probe of $250 \mu \mathrm{m}$ length, $50 \mu \mathrm{m}$ width, and $3 \mu \mathrm{m}$ thickness has a measured spring constant of $0.082 \mathrm{~N} / \mathrm{m}$. Lateral spatial resolution of $<50 \mathrm{~nm}$, topographic resolution of $<1 \mathrm{~nm}$ and thermal resolution of $<1.2 \mathrm{mK}$ are demonstrated. Wet scanning capability, which widens the possibility of biochemical applications, is also demonstrated for the first time. The structure, fabrication, and assembly of the probe and the interface circuit used for these experiments are described.
\end{abstract}

\section{INTRODUCTION}

We are developing ultra compliant thermal probes for critical applications in ULSI lithography research. These include, specifically, mapping the latent image of exposed but undeveloped photoresist (PR) to measure photo-acid generation and diffusion independently from the developing step [1]. Thermal probes have also been used for data storage and other applications [2,3]. Since they offer sub-surface mapping capability, thermal probes also facilitate studies of intra-cellular features in bio-related research. To fulfill our need for these applications, probes must have low spring constant to prevent damaging the soft materials, and provide spatial resolution $<100 \mathrm{~nm}$. In addition, to permit scanning in aqueous environments, complete electrical insulation is necessary. Wet aqueous scans are particularly challenging because of enhanced parasitic thermal losses, the need for complete electrical insulation, and the impact of surface tension on the ultra compliant probes. These requirements can not be fulfilled by using the commercially available wire probe which is made of bent bare wires, and has a high spring constant $(5 \mathrm{~N} / \mathrm{m})$, limited spatial resolution, and no particular isolation [4].

We have previously reported thermocouple or bolometer probes fabricated by 6-7 mask surface micromachining processes using polyimide as the shank material, and the application of these probes for temperature mapping, sub-surface imaging, and the measurement of glass transition temperature in photoresists $[5,6]$. This paper reports on applying these probes to ULSI lithography research, particularly for mapping the latent image of exposed but undeveloped PR to measure photo-acid generation (PAG) and diffusion independently. Wet scanning capability, which widens the possibility of biochemical applications, is also demonstrated for the first time. This paper also describes changes that have been made to older versions of this probe to help achieve these capabilities.

\section{FABRICATION}

The basic structure of the probe is similar to that reported in $[5,6]$, with a thin film bolometer sandwiched between two layers of polyimide, forming a cantilever. At one end of the cantilever the metal thin film protrudes through an opening in lower polyimide layer, where it is molded into a pyramidal tip by a notch that was anisotropically wet-etched into the substrate. The tip and a portion of the probe shank are then released from the substrate by etching an underlying sacrificial layer. The released length is then folded over to extend past the die edge for clearance and held in place by an adhesive (Fig. 1). Typical dimensions of the probes after assembly are $250 \mu \mathrm{m}$ length, $50 \mu \mathrm{m}$ width, and $3 \mu \mathrm{m}$ thickness with $\mathrm{Cr} / \mathrm{Au}(200 / 2000 \AA)$ for the tip and lead, which provides bolometer resistance about $60 \Omega$.

The present manifestation of the probe (Fig. 2) differs from the ones reported in the past $[5,6]$ in important ways. It includes a thin film of gold (Cr/Au: $200 / 4000 \AA$ ) which holds the flipped over probe by a thermocompression bond. This method retains the flatness of the probe and significantly increases yield. This layer also serves as a mirror to permit simultaneous operation of the probe as an AFM. The revised process also provides a tip diameter of about $50 \mathrm{~nm}$ by sharpening the notch used as its mold by nonuniform oxide growth [7]. For operation in aqueous environments, the bond pads and bond wires are covered with a thin layer of polyimide (PI2613 ${ }^{\mathrm{TM}}$ ), leaving the scanning tip as the only exposed metallic surface (Fig. 3).

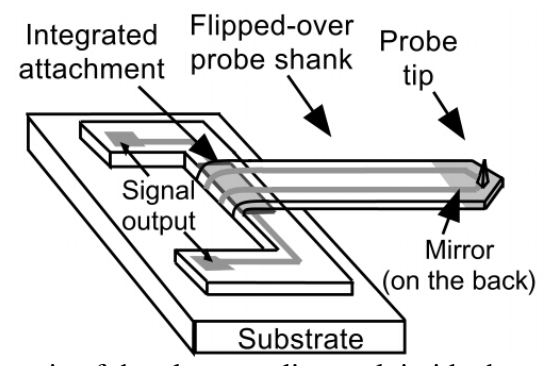

Fig. 1: Schematic of the ultracompliant polyimide thermal probe.

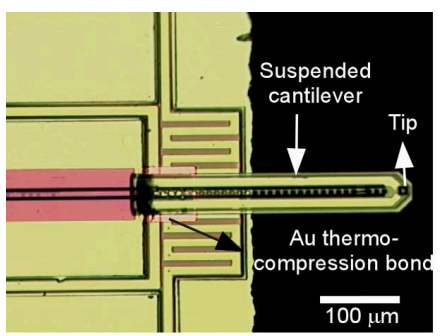

Fig. 2: A $250 \mu \mathrm{m}$ long probe flipped over the die edge and held down with a gold thermocompression bond.

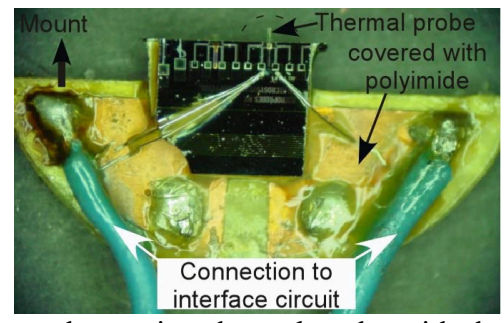

Fig. 3: A mounted scanning thermal probe with the base covered with a polyimide (PI2613 ${ }^{\mathrm{TM}}$ ) layer for working in aqueous environments. 


\section{OPERATION AND DESIGN OPTIMIZATION}

Figure 4 shows an interface circuit used to sense the probe resistance change as it scans across the sample surface. It includes a Wheatstone bridge, two gain stages providing combined amplification of $10^{4}$, and a low pass filter with cutoff frequency of $1 \mathrm{KHz}$ to reduce noise. The output voltage $\left(\mathrm{V}_{\text {out }}\right)$ is plotted in thermal scans.

The probe tip temperature change $\left(\Delta \mathrm{T}_{\mathrm{P}}\right)$ can be calculated from the output voltage change $\left(\Delta \mathrm{V}_{\text {out }}\right)$, which is:

$$
\Delta T_{P} \cong \Delta V_{\text {out }} \cdot\left(R_{1}+R_{P}\right)^{2} /\left(10^{4} \cdot V_{S} \cdot R_{1} \cdot R_{P} \cdot T C R\right)
$$

A $\Delta \mathrm{V}_{\text {out }}$ of $100 \mathrm{mV}$ corresponds to a $\Delta \mathrm{T}_{\mathrm{P}}$ of $5.657 \mathrm{mK}$ calculated using the voltage and resistance values shown in Fig. 4, and the measured temperature coefficient of resistance (TCR) of 3640 $\mathrm{ppm} / \mathrm{K}$ for the $\mathrm{Cr} / \mathrm{Au}$ bolometer. The full scale $\Delta \mathrm{T}_{\mathrm{p}}$ is indicated for all thermal scans presented in this paper. Before a scan, this circuit is adjusted to balance the Wheatstone bridge with the probe in contact with the sample by adjusting the control resistor $\left(R_{c}\right)$ to make the resistance ratio of $R_{c} / R_{2}$ equal to $R_{P} / R_{1}$ so that the output voltage is as close as possible to $0 \mathrm{~V}$.

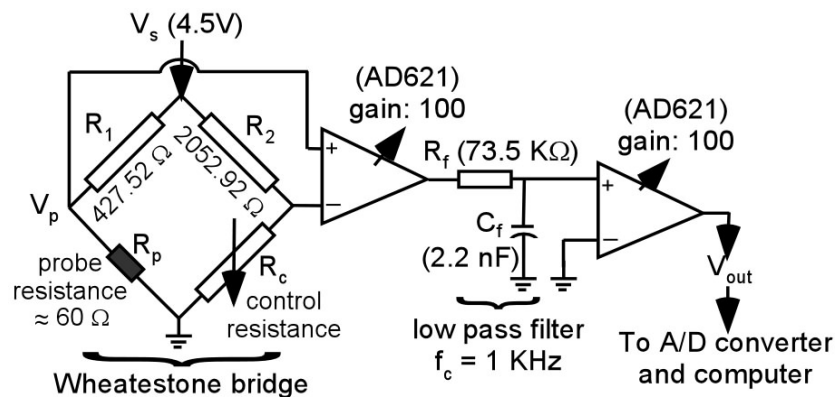

Fig. 4: An interface circuit for sensing the probe resistance change.

In scanning a $\mathrm{Si}$ wafer with an ultra-thin photoresist layer of thickness comparable to the scan tip diameter, the heat transfer between the probe tip and silicon substrate can be modeled as that through a cylinder. The heat loss $\left(\mathrm{P}_{\mathrm{s}}\right)$ to the silicon substrate can be expressed as:

$$
P_{s}=\left(T_{p}-T_{0}\right) \cdot A_{0} \cdot k_{s} / H
$$

where $\mathrm{T}_{\mathrm{p}}$ is the probe tip temperature, $\mathrm{A}_{0}$ is the tip-sample contact area, $\mathrm{k}_{\mathrm{s}}$ is the thermal conductivity of photoresist, and $\mathrm{H}$ is the photoresist thickness. The silicon substrate is a large heat sink, effectively with a fixed temperature $\mathrm{T}_{0}$. The thermal conductance image obtained from $\mathrm{V}_{\text {out }}$ contains both topographic and thermal conductivity information. A purely topographic image is produced simultaneously by monitoring the deflection of the probe cantilever as in a conventional AFM. It is often useful to compare these images for sub-surface and material property information.

\section{MEASUREMENT RESULTS}

The spring constant of a $250 \mu \mathrm{m}$ long, $50 \mu \mathrm{m}$ wide, and $3 \mu \mathrm{m}$ thick probe shank measured by using a built-in function in the Topometrix ${ }^{\mathrm{TM}}$ scanning system is $0.082 \mathrm{~N} / \mathrm{m}$, which is about $10 \times$ and $50 \times$ lower compared those reported in $[2,4]$. This allows contact mode scanning of soft materials such as photoresist patterns with feature size of $500 \mathrm{~nm}$ easily even without $z$-direction feedback (Fig. 5). The output voltage drops as the probe is scanned across the higher thermal conductivity material (silicon), because the heat loss from the tip to sample increases, which reduces the tip temperature, and the corresponding probe resistance.

Figure 6 shows the topographic and thermal images of exposed but undeveloped UV113 ${ }^{\mathrm{TM}}$ photoresist patterned with critical dimension of $70 \mathrm{~nm}$ and $200 \mathrm{~nm}$ pitch. Comparing the topographic and thermal images of partial developed UV $113^{\mathrm{TM}}$ photoresist sample (Fig. 7), it is evident that the thermal probe has a lateral spatial resolution of $<50 \mathrm{~nm}$. The topographical resolution is $<1 \mathrm{~nm}$. The interface circuit is sensitive enough to detect a tip temperature change only $1.13 \mathrm{mK}$.

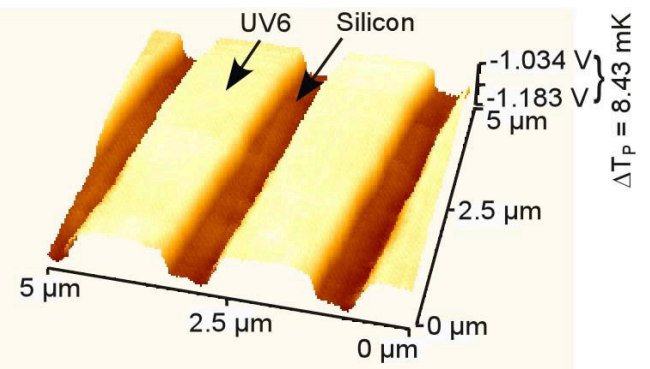

Fig. 5: Thermal image of developed $\mathrm{UV}^{\mathrm{TM}}$ photoresist sample with thickness of $350 \mathrm{~nm}$. No $z$-direction feedback.

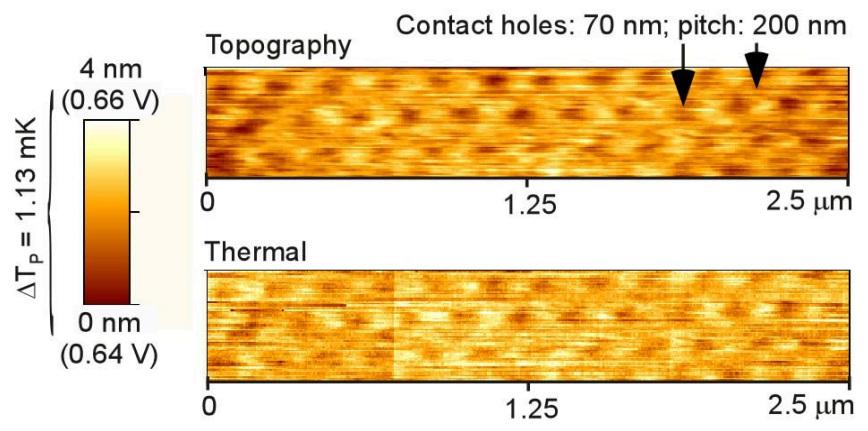

Fig. 6: Topographic (top) and thermal (bottom) images of exposed but undeveloped UV $113^{\mathrm{TM}}$ photoresist contact holes of $70 \mathrm{~nm}$.

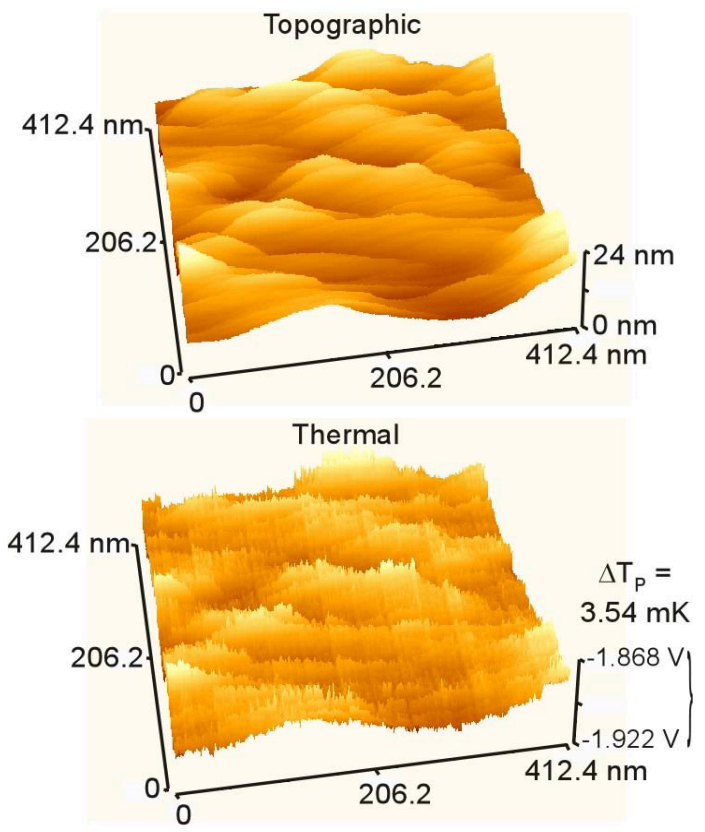

Fig. 7: Topographic (top) and thermal (bottom) images of partial developed UV113 ${ }^{\mathrm{TM}}$ photoresist sample show the thermal probe has spatial resolution of sub-50 $\mathrm{nm}$.

The positive tone chemically amplified photoresist $U V 6^{\mathrm{TM}}$ by Shipley, which is suitable for ultra-narrow ULSI linewidths, behaves as shown in Fig. 8 [8]. Unlike standard PR and PMMA, a photoacid generated by exposure permits thermolysis of the backbone polymer during the post exposure bake (PEB), which changes the solubility of the exposed regions of the resist and 
releases isobutylene. The photoresist thickness decreases in exposed areas where released isobutylene is evaporated during PEB. It is, therefore, important to control the PEB conditions to suppress the acid diffusion for critical dimension control when using chemical amplification resist systems.

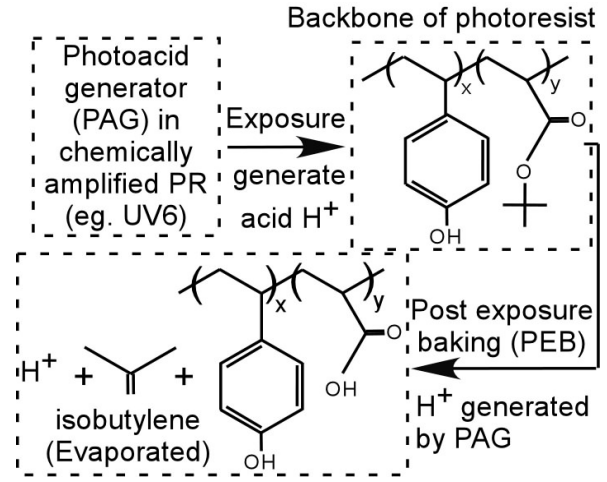

Fig. 8: In UV6 ${ }^{\mathrm{TM}}$, PAG acid deprotects the backbone during PEB for subsequent dissolution in the developer

Figure 9 shows the topographic and thermal linear scans of a trench in exposed but undeveloped Shipley UV6 ${ }^{\mathrm{TM}}$ photoresist. As the duration of the $130^{\circ} \mathrm{C}$ PEB increases from $45 \mathrm{~s}$ to $360 \mathrm{~s}$, both the topographic height change $(\Delta \mathrm{h})$ and $\Delta \mathrm{V}_{\text {out }}$ increase. However, the most significant change in $\Delta \mathrm{h}$ occurs in the 45-90 s period, whereas the most significant change in $\Delta \mathrm{V}_{\text {out }}$ occurs in the 180-360 $\mathrm{s}$ period. As noted above, the $\Delta \mathrm{h}$ is due to the release of isobutylene and depends on the acid concentration and the reaction rate of photoresist backbone deprotection. The "v" shape profiles of exposed regions are due to the Gaussian distribution of the ebeam source of dose profile, and hence, the acid concentration [1]. As the PEB time increases, the " $v$ " shape profile widens due to the acid diffusion. However, since the acid diffusion constant, which depends on the process conditions [9], is only about $50 \mathrm{~nm}^{2} / \mathrm{s}$ for $\mathrm{UV}^{\mathrm{TM}}[10]$, this change is very small $(\sim 10 \mathrm{~nm})$ [11], particularly when compared to the $500 \mathrm{~nm}$ width of the exposed portion. Since the recommended PEB condition for $130{ }^{\circ} \mathrm{C}$ is $90 \mathrm{~s}$, the longer PEB times may deplete the photoresist backbone, minimizing subsequent changes in topography. In contrast, other chemical changes may lead to the $\Delta \mathrm{V}_{\text {out }}$ increase during the $180-360 \mathrm{~s}$ period.

The thermal probe was also used to scan HeLa line ovarian cancer tumor cells which are widely used in cell research [12]. The optical and thermal images of the nucleus of a HeLa cell fixed to a glass slide while undergoing mitosis are compared in Fig. 10. Since the topographic variation in such a sample is much larger than the thermal conductivity difference of the organelles within it, the thermal image is very similar to the topography map. Comparing the topographic (AFM) and thermal images of a layer of HeLa cells (Fig. 11), it is evident that the thermal image can provide more details.

Figure 12 shows thermal 2D and linear scans of metal lines on a $\mathrm{Si}$ substrate in an aqueous environment. Deformation of the ultracompliant probe by meniscus forces is prevented by immersing the entire mounting platform in the water along with the sample. The variation in metal films is clearly detectable despite the fact that these scans were performed without $z$-axis feedback. This is possible because of the ultra compliant nature and high thermal isolation offered by the probe. Operation in aqueous environments facilitates observation of metabolic activity in living cells.
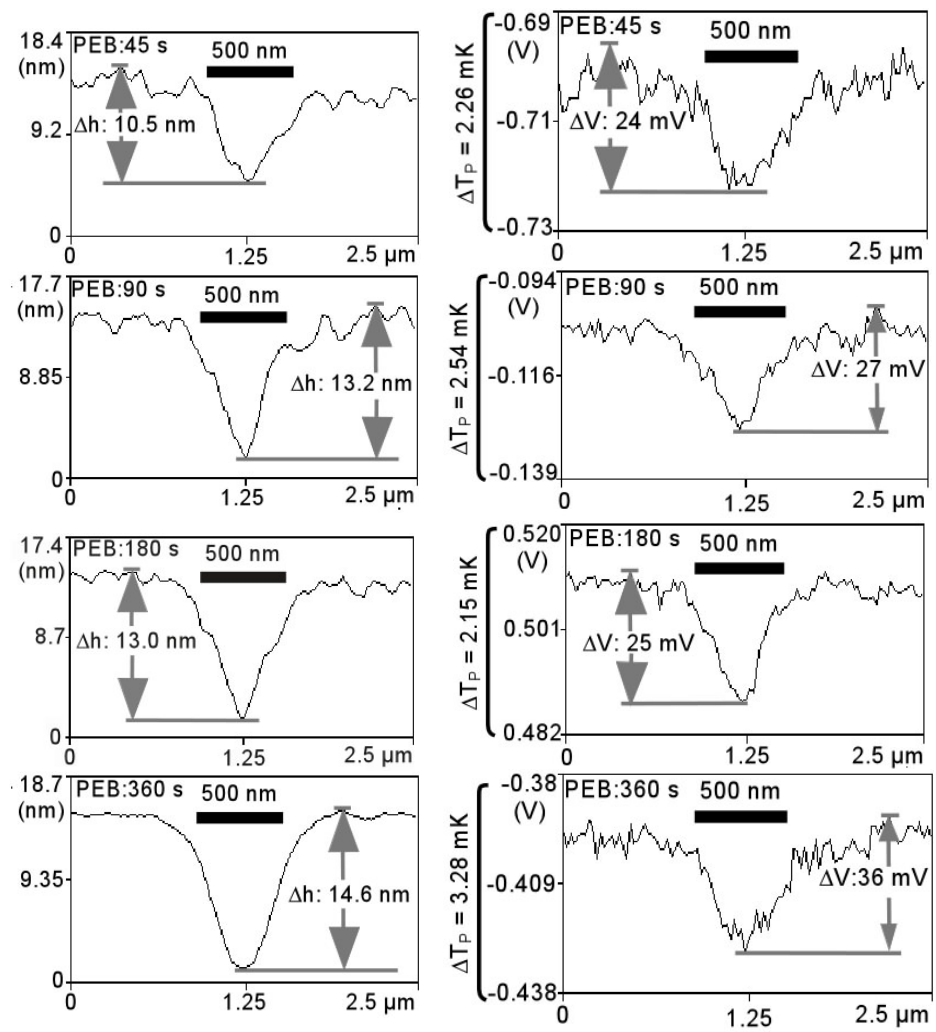

Fig. 9: Topographic (left) and thermal (right) line scans of a latent image in $\mathrm{UV}^{\mathrm{TM}}$ with varying PEB time at $130^{\circ} \mathrm{C}$.
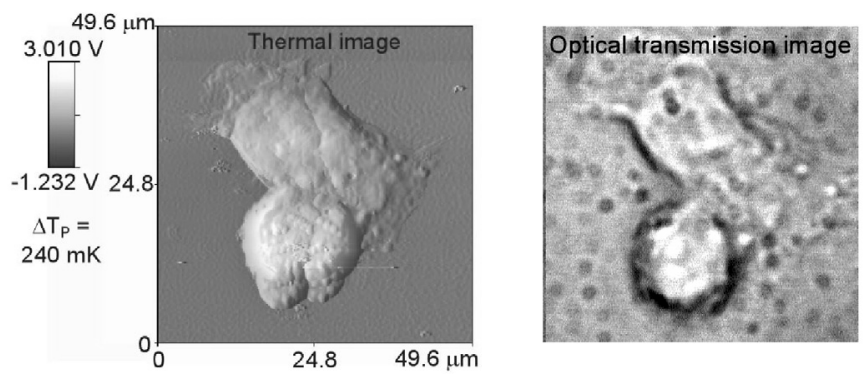

Fig. 10: Thermal (left) and optical (right) images of the nuclei HeLa tumor cells on a glass slide.

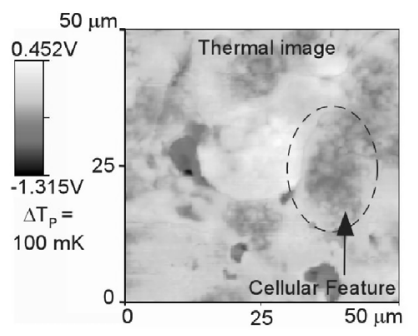

Fig. 11: Thermal (upper) and topographic (lower) images of a layer of HeLa tumor cell on a

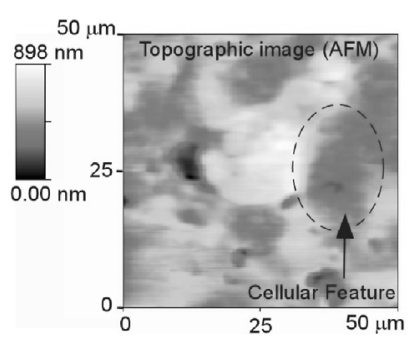
glass cover slide. The thermal image shows more cellular details. 

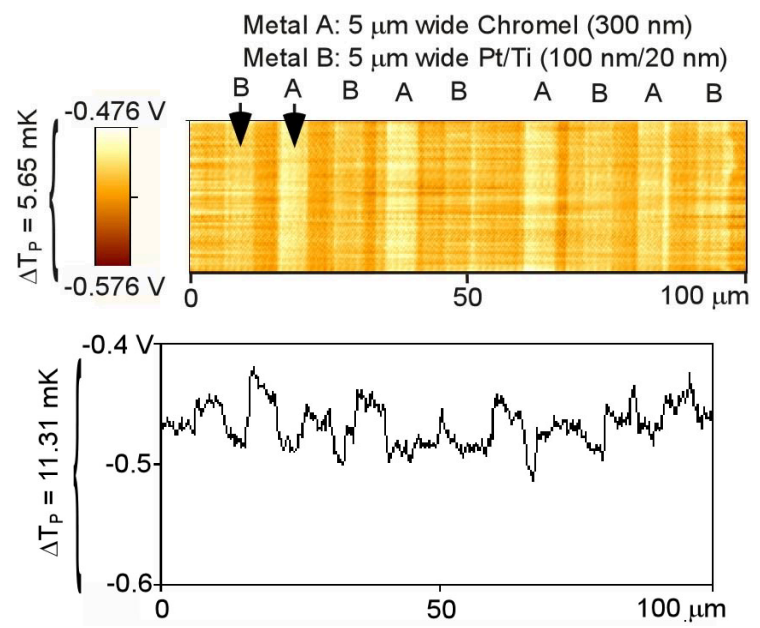

Fig. 12: Area (upper) and linear (lower) thermal scans of metal stripes on a Si substrate obtained without $z$-direction feedback with the probe and sample immersed in water.

\section{CONCLUSION}

This effort has addressed the development and applications of a polyimide shank thermal probe fabricated by a 6-mask process. These probes are assembled with the help of a thermocompression bond between thin films that greatly improves yield. A coating of polyimide over the pads and wire bonds after assembly permits its operation in aqueous environments. Typical probe dimensions are $250 \mu \mathrm{m}$ length, $50 \mu \mathrm{m}$ width, and $3 \mu \mathrm{m}$ thickness. The probe is ultra-compliant with a spring constant of $0.082 \mathrm{~N} / \mathrm{m}$, which can be further reduced by changing the material and/or dimensions. It can be operated without $z$-direction feedback, even when scanning soft materials. The probe offers lateral spatial resolution of $<50 \mathrm{~nm}$, topographical resolution of $<1 \mathrm{~nm}$, and tip temperature resolution of $<1.2 \mathrm{mK}$. The probe has been used to scan exposed but undeveloped photoresist samples, to study the acid diffusion in photoresist during post-exposure bake, and to image fixed HeLa cells. Scans of thin film metals demonstrated its usefulness while operating in a liquid medium. A summary of its operating limits is presented in Table I.

Table I: Performance summary for the polyimide probe.

\begin{tabular}{|c|c|}
\hline Tip Diameter & $\approx 50 \mathrm{~nm}$ \\
\hline Lateral Spatial Resolution & $<50 \mathrm{~nm}$ \\
\hline Topographical resolution & $<1 \mathrm{~nm}$ \\
\hline Input Referred Circuit Noise (1 KHz BW) & $<2 \mu \mathrm{V}$ \\
\hline$\Delta$ R Resolution & $<0.25 \mathrm{~m} \Omega$ \\
\hline Tip Temperature Resolution & $<1.2 \mathrm{mK}$ \\
\hline Detectable Thermal Conductance Change & $1 \times 10^{-11} \mathrm{~W} / \mathrm{K}(1 \%)$ \\
\hline Detectable Thermal Conductivity Change & $2 \times 10^{-3} \mathrm{~W} / \mathrm{m} \cdot \mathrm{K}(1 \%)$ \\
\hline
\end{tabular}

\section{ACKNOWLEDGEMENTS}

The authors thank Dr. Leo Ocola for supporting the UV113 ${ }^{\mathrm{TM}}$ photoresist sample, and Dr. Saulius Vainauskas for providing HeLa cell samples. This work was funded in part by the Semiconductor Research Corporation contract \# 98-LP-452.005. The Center for NanoTechnology, University of Wisconsin-Madison, is supported in part by DARPA/ONR grant \# MDA 972-00-1-0018, and \# MDA 972-99-1-0013. The Synchrotron Radiation Center, University of Wisconsin-Madison, at which some of the experimental facilities are located, is operated under NSF award \# DMR-0084402. Partial travel support was provided by the Transducers Research Foundation and the DARPA MEMS and BioFlips programs.

\section{REFERENCES}

[1] L.E.Ocola, D.Fryer, P.Nealey, J.dePablo, F.Cerrina, and S. Kämmer, "Latent image formation: Nanoscale topography and calorimetric measurements in chemically amplified resists," $J$. Vac. Sci. Technol. B, 14(6), pp. 3974-9, 1996.

[2] P.Vettiger, et al., "The "Millipede"-More than one thousand tips for future AFM data storage," IBM J. Res. Develop., 44(3), pp. 323-40, 2000.

[3] A.Majumdar, "Scanning thermal microscopy," Annu. Rev. Mater. Sci., 29, pp. 505-85, 1999.

[4] TM Microscopes, www.tmmicro.com, probe model\# 1615-00.

[5] M.-H.Li, J.J.Wu, Y.B.Gianchandani, "Surface micromachined polyimide scanning thermocouple probes," $J$. Microelectromech. Sys., 10(1), pp. 3-9, 2001.

[6] M.-H. Li, Y.B. Gianchandani, "Microcalorimetry applications of a surface micromachined bolometer-type thermal probe," $J$. Vac. Sci.Technol. B, 18(6), pp. 3600-3, 2000.

[7] S.Akamine, C.F.Quate, Low temperature thermal oxidation sharpening of microcast tips, J. Vac. Sci. Technol. B, 10(5), pp. 2307-10, 1992.

[8] H.Ito, "Chemically amplified resists: past, present, and future," SPIE Vol. 3678, pp. 2-12, 1999.

[9] T.Itani, H.Yoshino, S.Hashimoto, M.Yamana, N.Samoto, K. Kasama, "A study of acid diffusion in chemically amplified deep ultraviolet resist," J. Vac. Sci. Technol. B, 14(6), pp. 4226-8, 1996.

[10] D.Kang, E.K.Pavelchek, C.Swible-Keane, "The accuracy of current model descriptions of a DUV phtotresist," SPIE, vol. 3678, pp. 877-90, 1999.

[11] T.H.Fedynyshyn, J.W.Thackeray, J.H.Georger, M.D. Denison, "Effect of acid diffusion on performance in positive deep ultraviolet resists," J. Vac. Sci. Technol. B, 12(6), pp. 3888-94, 1994.

[12] R.L.Warters, O.L.Stone, "Histone protein \& DNA synthesis in HeLa cells after thermal shock," J. Cell. Phys., 118, pp. $153-$ 60, 1984. 